SSU-HEP-12/07

\title{
Relativistic corrections to double charmonium production in high energy proton-proton interaction
}

\author{
A.P. Martynenkd* \\ Samara State University, Pavlov Street 1, 443011, Samara, Russia and \\ Samara State Aerospace University named after S.P. Korolyov, \\ Moskovskoye Shosse 34, 443086, Samara, Russia \\ A.M. Trunin讨 \\ Samara State Aerospace University named after S.P. Korolyov, \\ Moskovskoye Shosse 34, 443086, Samara, Russia
}

\begin{abstract}
On the basis of pertubative QCD and the relativistic quark model we calculate relativistic corrections to the process of pair $J / \psi$ production in proton-proton collisions at LHC energy $\sqrt{S}=7 \mathrm{TeV}$. Relativistic terms in the production amplitude connected with the relative motion of heavy quarks and the transformation law of the bound state wave functions to the reference frame of moving $J / \psi$ mesons are taken into account. For the gluon and quark propagators entering the amplitude we use a truncated expansion in relative quark momenta up to the second order. Relativistic corrections to the quark bound state wave functions are considered by means of the Breit-like potential. It turns out that the examined effects decrease initial nonrelativistic cross section more than two times. The final result lies below the experimental value measured by $\mathrm{LHCb}$.
\end{abstract}

PACS numbers: 13.66.Bc, 12.39.Ki, 12.38.Bx

Keywords: Hadron production in proton-proton interaction, Relativistic quark model

\section{INTRODUCTION}

The production of heavy quarkonium states in different reactions is the subject of considerable interest during last years. The mechanism of heavy quarkonium production represents the long-standing problem of quantum chromodynamics [1-4]. Most current theoretical investigations are performed on the basis of nonrelativistic quantum chromodynamics (NRQCD) [5] and the quark models. According to these approaches the production of heavy quarkonium is divided into two stages. On the first stage one or several quark-antiquark pairs are produced at small distances of order $1 / m_{Q}$. This short-range part in the production is associated with the basic interaction of free quarks and gluons and can be evaluated

*Electronic address: a.p.martynenko@samsu.ru

†Electronic address: amtrnn@gmail.com 
by perturbation theory. The subsequent nonperturbative transition from the intermediate states of quarks $Q Q \ldots$ and antiquarks $\bar{Q} \bar{Q} \ldots$ to physical quarkonium states, on the second stage, involves long-distance scales of order of quarkonium size $1 /\left(m_{Q} v\right)$. The formation of the quark bound states is parameterized by nonperturbative matrix elements in NRQCD and calculated by means of the bound state wave functions in the quark models. The finding of the correspondence between parameters of the quark models and NRQCD opens the way for better understanding of the quark-gluon dynamics. Both approaches complement each other and can reveal new aspects of the color dynamics of quarks and gluons.

One of the directions in this investigation is related with the pair production of double heavy quarkonium. The initial impulse to intensive investigations was given in this field several years ago by the measurement of the pair charmonium production cross sections in electron-positron annihilation. The experimental data obtained at the Belle and BaBar detectors disagreed with the calculations based on NRQCD. The theoretical results were improved and adjusted in correspondence with the experiment after the account of one-loop perturbative corrections and relativistic corrections to the nonrelativistic cross section 6 8]. One of the learned lessons from this problem consists in the understanding that only sequential relativistic approach to the heavy quarkonium production processes can lead to reliable theoretical results. It is necessary to point out that subsequent observation of numerous charmonium-like states by the Belle and BaBar collaborations with unclear nature demands further continuation of the investigations in this direction [9]. Recently, the first experimental result of the $\mathrm{LHCb}$ collaboration on the pair charmonium production in proton-proton interaction was published [10]:

$$
\sigma_{L H C b}^{e x p}=5.1 \pm 1.0 \pm 1.1 \mathrm{nb},
$$

where the first uncertainty is statistical and the second systematic. It agrees with the theoretical estimation of the cross section $\sigma=17 \div 22 \mathrm{nb}$ obtained in the leading order of QCD where the process of the gluon fusion is the dominant one [11 15]. These calculations give the total value of the cross section for the pair charmonium production $\sigma=3 \div 5 \mathrm{nb}$ in the kinematical region of the LHCb experiment (the region of rapidities $2<y<4.5$ ). The theoretical uncertainty remains sufficiently large. To the present the calculations of the pair charmonium production in $p p$ interaction were carried out in the leading order of QCD without inclusion of relativistic corrections. In addition to these permanent theoretical errors known from the experience of $e^{+} e^{-}$annihilation we have in this task new specific uncertainty caused by parton distribution functions at small $x$ values because the gluon contribution from the region of small $x$ is dominant. There appears also another uncertainty related with the double parton interaction [16]. In this work we study one aspect of the improvement of the previous calculations connected with the account of relativistic corrections. Using the methods of the relativistic quark model [8, 17-19] we perform new calculation of the cross section $\sigma(p p \rightarrow J / \psi J / \psi)$ accounting for relativistic corrections to the production amplitude and the bound state wave functions of heavy $c$-quarks. So, the aim of this study consists in the relativistic description of the pair charmonium production at hadron colliders. It is important to note that the interest to the inclusive reactions $p+p \rightarrow 2 J / \psi+X$, $p N \rightarrow 2 J / \psi+X$ is not limited only by the investigation of the production mechanism. The study of the quarkonium production in the nuclear matter leads to new data about QCD at high density and temperature [20, 21].

There exist different mechanisms for the pair charmonium production in $p p$-collisions. At the collider energies, double quarkonium production occurs through the gluon-gluon 
interaction channel. In the color singlet model (CSM) a pair of quark-antiquark $(c \bar{c})$ is created at short distances in color singlet state. Then it evolves nonperturbatively into an observed meson $J / \psi$. At small transverse momenta and small invariant masses of the $J / \psi$ pair the color singlet mechanism gives the main contribution to the cross section. Another possibility is to create a pair $(c \bar{c})$ with different color and angular momentum from that of the final meson. Then the color octet pair evolves to the color singlet charmonium emitting soft gluons. This color octet mechanism (COM) plays significant role in the region of high transverse momentum. Among large number of the Feynman diagrams describing the production of a pair $J / \psi$ it has been separated a class in which the $J / \psi$ pair production is related with the double gluon fragmentation. In this study we analyze the total set of the production amplitudes in the color singlet model making primary emphasis upon relativistic effects.

\section{GENERAL FORMALISM}

The differential cross section $d \sigma$ for the inclusive double charmonium production in proton-proton interaction can be presented in the form of the convolution of partonic cross section $d \sigma(g g \rightarrow J / \psi J / \psi)$ with the parton distribution functions (PDF) in the initial protons [1, 2]:

$$
d \sigma[p+p \rightarrow J / \psi+J / \psi+X]=\int d x_{1} d x_{2} f_{g / p}\left(x_{1}, \mu\right) f_{g / p}\left(x_{2}, \mu\right) d \sigma[g g \rightarrow J / \psi J / \psi],
$$

where $f_{g / p}(x, \mu)$ is partonic distribution function for the gluon in the proton, $x_{1,2}$ are longitudinal momentum fractions of gluons, $\mu$ is the factorization scale. Neglecting the proton mass and taking the c.m. reference frame of initial protons with the beam along the $z$ axis we can present the gluon on mass-shell momenta $k_{1,2}=x_{1,2} \frac{\sqrt{S}}{2}(1,0,0, \pm 1) . \sqrt{S}$ is the center-of-mass energy in proton-proton collision.

In the quasipotentional approach the invariant transition amplitude for the gluonic subprocess $g+g \rightarrow J / \psi+J / \psi$ can be presented as a convolution of a perturbative production amplitude of two $c$-quark and $\bar{c}$-antiquark pairs $\mathcal{T}\left(p_{1}, p_{2} ; q_{1}, q_{2}\right)$ and the quasipotential wave functions of the final mesons $\Psi_{J / \psi}[8]$ :

$$
\mathcal{M}[g g \rightarrow J / \psi J / \psi]\left(k_{1}, k_{2}, P, Q\right)=\int \frac{d \mathbf{p}}{(2 \pi)^{3}} \int \frac{d \mathbf{q}}{(2 \pi)^{3}} \bar{\Psi}(p, P) \bar{\Psi}(q, Q) \otimes \mathcal{T}\left(p_{1}, p_{2} ; q_{1}, q_{2}\right),
$$

where $p_{1}$ and $p_{2}$ are four-momenta of $c$-quark and $\bar{c}$-antiquark in the pair forming the first $J / \psi$ particle, and $q_{2}$ and $q_{1}$ are appropriate momenta for quark and antiquark in the second meson $J / \psi$. They are defined in terms of total momenta $P(Q)$ and relative momenta $p(q)$ as follows:

$$
p_{1,2}=\frac{1}{2} P \pm p, \quad(p P)=0 ; \quad q_{1,2}=\frac{1}{2} Q \pm q, \quad(q Q)=0,
$$

In Eq. (3) we integrate over the relative three-momenta of quarks and antiquarks in the final state. The systematic account of all terms depending on the relative quark momenta $p$ and $q$ in (2) is important for increasing the accuracy of the calculation. $p=L_{P}(0, \mathbf{p})$ and $q=L_{Q}(0, \mathbf{q})$ are the relative four-momenta obtained by the Lorentz transformation of fourvectors $(0, \mathbf{p})$ and $(0, \mathbf{q})$ to the reference frames moving with the four-momenta $P$ and $Q$. 

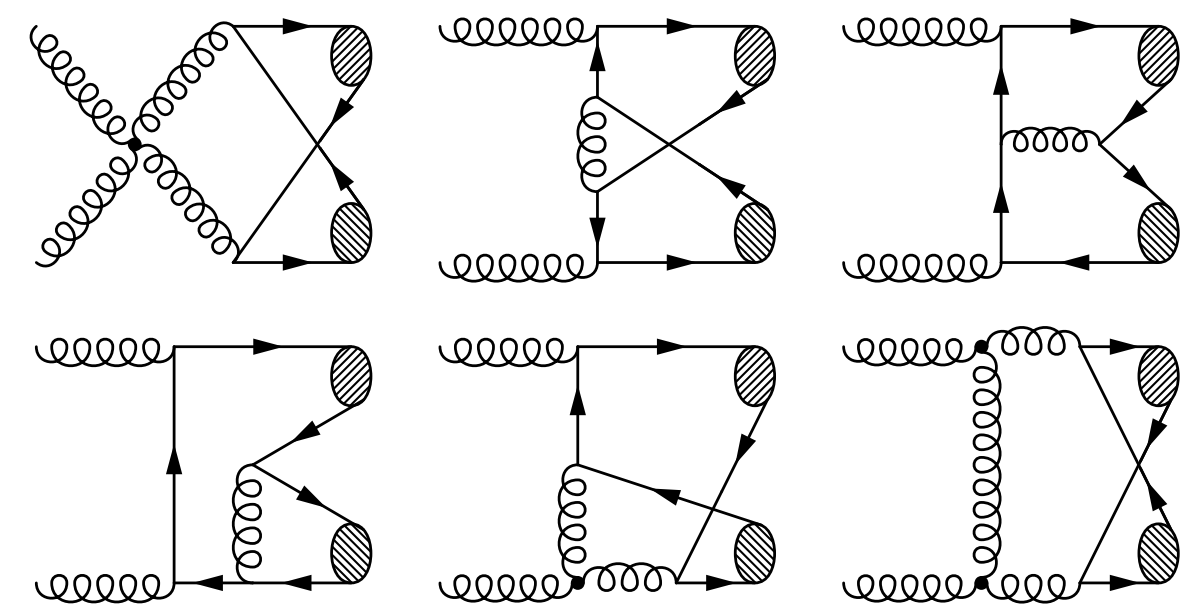

FIG. 1: The typical LO diagrams contributing to the partonic process $g+g \rightarrow J / \psi+J / \psi$. The others can be obtained by reversing the quark lines or interchanging the initial gluons.

The parton-level differential cross section for $g+g \rightarrow J / \psi+J / \psi$ is expressed further through the Mandelstam variables $s, t$ and $u$ :

$$
s=\left(k_{1}+k_{2}\right)^{2}=(P+Q)^{2}=x_{1} x_{2} S,
$$

$t=\left(P-k_{1}\right)^{2}=\left(Q-k_{2}\right)^{2}=M^{2}-x_{1} \sqrt{S}\left(P_{0}-|\mathbf{P}| \cos \phi\right)=M^{2}-x_{1} x_{2} S+x_{2} \sqrt{S}\left(P_{0}+|\mathbf{P}| \cos \phi\right)$, $u=\left(P-k_{2}\right)^{2}=\left(Q-k_{1}\right)^{2}=M^{2}-x_{2} \sqrt{S}\left(P_{0}+|\mathbf{P}| \cos \phi\right)=M^{2}-x_{1} x_{2} S+x_{1} \sqrt{S}\left(P_{0}-|\mathbf{P}| \cos \phi\right)$,

where $M$ is the charmonium mass, $\phi$ is the angle between $\mathbf{P}$ and the $z$-axis. The transverse momentum $P_{T}$ of $J / \psi$ and its energy $P_{0}$ can be written as

$$
P_{T}^{2}=|\mathbf{P}|^{2} \sin ^{2} \phi=-t-\frac{\left(M^{2}-t\right)^{2}}{x_{1} x_{2} S}, \quad P_{0}=\frac{x_{1} x_{2} \sqrt{S}}{x_{1}+x_{2}}+\frac{x_{1}-x_{2}}{x_{1}+x_{2}}|\mathbf{P}| \cos \phi .
$$

At leading order of perturbation theory in strong coupling constant $\alpha_{s}$ there are 31 Feynman diagrams contributing to the amplitude of pair $J / \psi$ production due to gluon fusion. The typical diagrams from this set are presented in Fig. 1. For the completeness we show in Figs. 23 also the Feynman diagrams from two other subsets containing 5 and 8 Feynman diagrams which do not contribute to the production amplitude. Any Feynman amplitude shown in Fig. 2 has zero contribution because its color factor is equal to zero. The sum of four diagrams from the subset in Fig. 3 is equal zero with the account of relativistic corrections studied in this work. So, further we consider only the 31 Feynman amplitudes from Fig. 1.

Let us consider, for example, the transformation of the first amplitude in Fig. 1 which takes the form:

$$
\begin{gathered}
\mathcal{T}_{1}^{a b}\left(p_{1}, p_{2} ; q_{1}, q_{2}\right)=8 \pi^{2} \alpha_{s}^{2} \delta^{a b} \varepsilon_{1}^{\lambda}\left(k_{1}\right) \varepsilon_{2}^{\mu}\left(k_{2}\right) \frac{2 g_{\lambda \mu} g_{\nu \sigma}-g_{\lambda \sigma} g_{\mu \nu}-g_{\lambda \nu} g_{\mu \sigma}}{\left(\frac{P}{2}+\frac{Q}{2}+p+q\right)^{2}\left(\frac{P}{2}+\frac{Q}{2}-p-q\right)^{2}} \times \\
{\left[\bar{u}\left(p_{1}\right) \gamma^{\sigma} v\left(q_{1}\right)\right]\left[\bar{u}\left(q_{2}\right) \gamma^{\nu} v\left(p_{2}\right)\right]}
\end{gathered}
$$


where $\varepsilon_{1}\left(k_{1}\right)$ and $\varepsilon_{2}\left(k_{2}\right)$ are the polarization vectors of initial gluons. The amplitude (3) should be convoluted with two wave functions of $J / \psi$ mesons taking in the reference frame moving with four momenta $P$ and $Q$. The transformation law of the bound state wave function from the rest frame to the moving one with four momentum $P$ was derived in the Bethe-Salpeter approach in Ref. [22] and in the quasipotential method in Ref. [23]. We use the last one and write the necessary transformation as follows:

$$
\begin{gathered}
\Psi_{P}^{\rho \omega}(\mathbf{p})=D_{1}^{1 / 2, \rho \alpha}\left(R_{L_{P}}^{W}\right) D_{2}^{1 / 2, \omega \beta}\left(R_{L_{P}}^{W}\right) \Psi_{0}^{\alpha \beta}(\mathbf{p}), \\
\bar{\Psi}_{P}^{\lambda \sigma}(\mathbf{p})=\bar{\Psi}_{0}^{\varepsilon \tau}(\mathbf{p}) D_{1}^{+1 / 2, \varepsilon \lambda}\left(R_{L_{P}}^{W}\right) D_{2}^{+1 / 2, \tau \sigma}\left(R_{L_{P}}^{W}\right),
\end{gathered}
$$

where $R^{W}$ is the Wigner rotation, $L_{P}$ is the Lorentz boost from the meson rest frame to a moving one, and the rotation matrix $D^{1 / 2}(R)$ is defined by

$$
\left(\begin{array}{ll}
1 & 0 \\
0 & 1
\end{array}\right) D_{1,2}^{1 / 2}\left(R_{L_{P}}^{W}\right)=S^{-1}\left(\mathbf{p}_{1,2}\right) S(\mathbf{P}) S(\mathbf{p}),
$$

where the explicit form for the Lorentz transformation matrix of the four-spinor is

$$
S(\mathbf{p})=\sqrt{\frac{\epsilon(p)+m}{2 m}}\left(1+\frac{(\boldsymbol{\alpha} \mathbf{p})}{\epsilon(p)+m}\right) .
$$

Omitting a number of transformations which can be performed with (7) in (3) as in [8, 18] we write the contribution to the production amplitude in the form:

$$
\begin{aligned}
& \mathcal{M}_{1}^{a b}\left(k_{1}, k_{2}, P, Q\right)=2 M \delta^{a b} \pi^{2} \alpha_{s}^{2} \int \frac{d \mathbf{p}}{(2 \pi)^{3}} \frac{\bar{\Psi}_{0}^{J / \psi}(\mathbf{p})}{\left[\frac{\epsilon(p)}{m} \frac{\epsilon(p)+m}{2 m}\right]} \int \frac{d \mathbf{q}}{(2 \pi)^{3}} \frac{\bar{\Psi}_{0}^{J / \psi}(\mathbf{q})}{\left[\frac{\epsilon(q)}{m} \frac{\epsilon(q)+m}{2 m}\right]} \times \\
& \operatorname{Tr}\left\{\left[\frac{\hat{v}_{1}-1}{2}+\hat{v}_{1} \frac{\mathbf{p}^{2}}{2 m(\epsilon(p)+m)}-\frac{\hat{p}}{2 m}\right] \hat{\varepsilon}_{P}^{*}\left(\hat{v}_{1}+1\right)\left[\frac{\hat{v}_{1}+1}{2}+\hat{v}_{1} \frac{\mathbf{p}^{2}}{2 m(\epsilon(p)+m)}+\frac{\hat{p}}{2 m}\right] \gamma^{\sigma} \times\right. \\
& \left.\left[\frac{\hat{v}_{2}-1}{2}+\hat{v}_{2} \frac{\mathbf{q}^{2}}{2 m(\epsilon(q)+m)}+\frac{\hat{q}}{2 m}\right] \hat{\varepsilon}_{Q}^{*}\left(\hat{v}_{2}+1\right)\left[\frac{\hat{v}_{2}+1}{2}+\hat{v}_{2} \frac{\mathbf{q}^{2}}{2 m(\epsilon(q)+m)}-\frac{\hat{q}}{2 m}\right] \gamma^{\nu}\right\} \times \\
& \times \varepsilon_{1}^{\lambda}\left(k_{1}\right) \varepsilon_{2}^{\mu}\left(k_{2}\right) \frac{2 g_{\lambda \mu} g_{\nu \sigma}-g_{\lambda \sigma} g_{\mu \nu}-g_{\lambda \nu} g_{\mu \sigma}}{\left(\frac{P}{2}+\frac{Q}{2}+p+q\right)^{2}\left(\frac{P}{2}+\frac{Q}{2}-p-q\right)^{2}},
\end{aligned}
$$

where $v_{1}=P / M, v_{2}=Q / M ; \varepsilon_{P, Q}$ are polarization vectors of final $J / \psi$ mesons with $\varepsilon_{P} \cdot P=$ 0 and $\varepsilon_{Q} \cdot Q=0 ; \epsilon(p)=\sqrt{m^{2}+\mathbf{p}^{2}}, M=2 m$ is the mass of $J / \psi$ meson. The hat symbol means contraction of the four-vector with the Dirac gamma-matrices. The spin projectors $v(0) \bar{u}(0)=\hat{\varepsilon}^{*}\left(1+\gamma_{0}\right) /(2 \sqrt{2})$ corresponding to $J / \psi$ mesons are introduced as well as projectors $\delta_{i j} / \sqrt{3}$ onto a color singlet states. We explicitly extracted in (11) the normalization factors $\sqrt{2 M}$ of quasipotential bound state wave functions.

The same transformations can be carried out with all 31 Feynman amplitudes in Fig. 1 In view of large volume of the calculations we have used the package FeynArts [24] for the system Mathematica and Form [25]. To make the entry of final amplitude more compact, we introduce a number of vertex functions $\Gamma_{i}$. Then we can present the total amplitude (3) in the form:

$$
\mathcal{M}[g g \rightarrow J / \psi J / \psi]\left(k_{1}, k_{2}, P, Q\right)=\frac{1}{9} M \pi^{2} \alpha_{s}^{2} \int \frac{d \mathbf{p}}{(2 \pi)^{3}} \int \frac{d \mathbf{q}}{(2 \pi)^{3}} \operatorname{Tr} \mathfrak{M},
$$



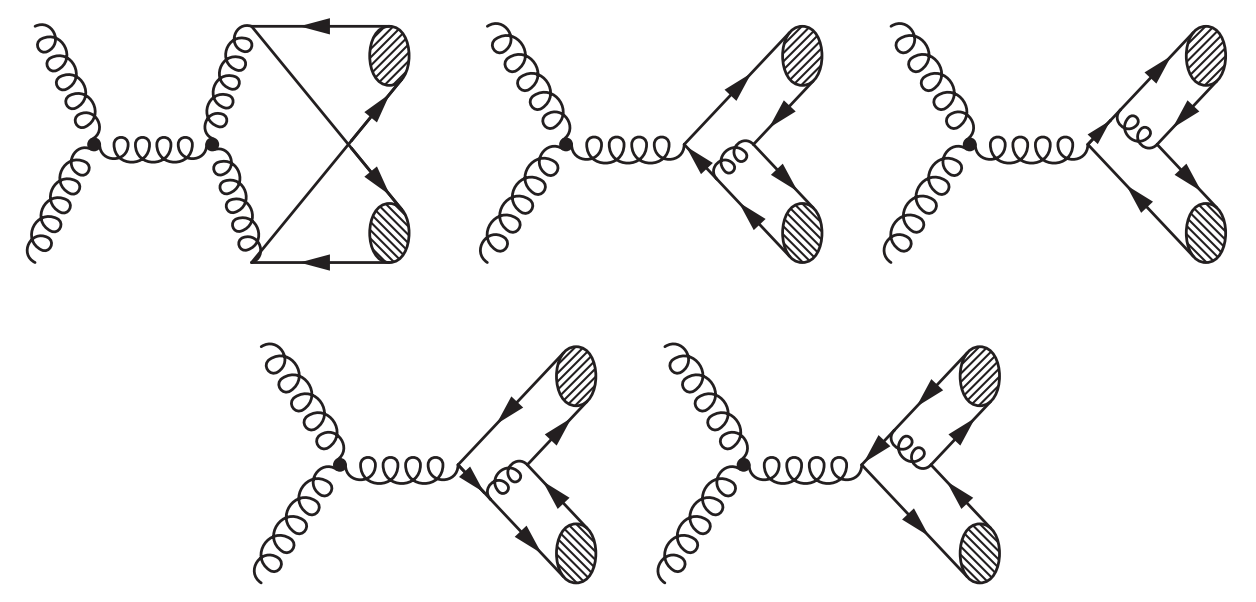

FIG. 2: The subset of LO diagrams which give zero contribution to $g+g \rightarrow J / \psi+J / \psi$ because their color factor is equal zero.
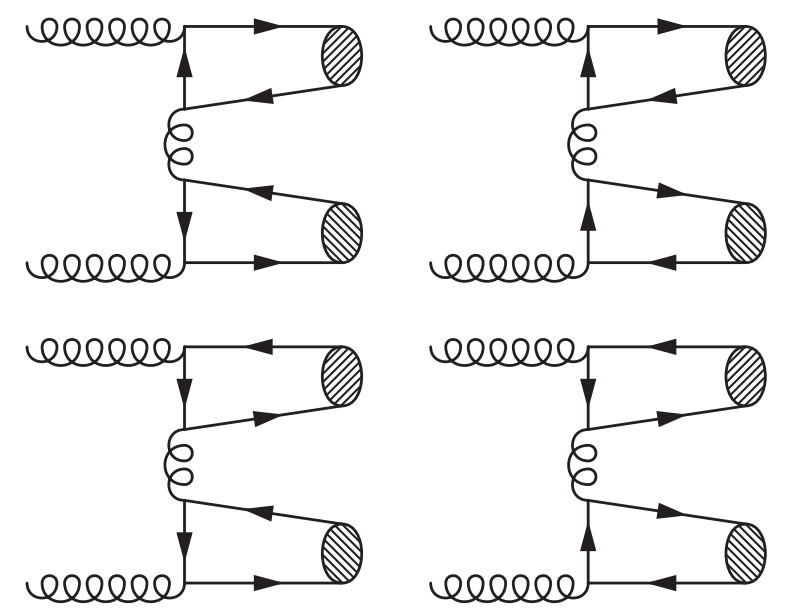

FIG. 3: The sum of LO diagrams from this subset gives zero contribution to $g+g \rightarrow J / \psi+J / \psi$ with the account of relativistic corrections of order $O\left(v^{2}\right)$.

$$
\begin{gathered}
\mathfrak{M}=\mathcal{D}_{1} \gamma_{\beta} \bar{\Psi}_{q, Q} \Gamma_{1}^{\beta} \bar{\Psi}_{p, P} \hat{\varepsilon}_{2} \frac{m-\hat{k}_{2}+\hat{p}_{1}}{\left(k_{2}-p_{1}\right)^{2}-m^{2}}+\mathcal{D}_{2} \gamma_{\beta} \bar{\Psi}_{q, Q} \Gamma_{2}^{\beta} \bar{\Psi}_{p, P} \hat{\varepsilon}_{1} \frac{m-\hat{k}_{1}+\hat{p}_{1}}{\left(k_{1}-p_{1}\right)^{2}-m^{2}}+ \\
\mathcal{D}_{3} \bar{\Psi}_{q, Q} \Gamma_{3}^{\beta} \bar{\Psi}_{p, P} \gamma_{\beta}+\mathcal{D}_{4} \bar{\Psi}_{p, P} \Gamma_{4}^{\beta} \bar{\Psi}_{q, Q} \gamma_{\beta}+\mathcal{D}_{1} \bar{\Psi}_{q, Q} \Gamma_{5}^{\beta} \bar{\Psi}_{p, P} \gamma_{\beta} \frac{m+\hat{k}_{2}-\hat{q}_{1}}{\left(k_{2}-q_{1}\right)^{2}-m^{2}} \hat{\varepsilon}_{2}+ \\
\mathcal{D}_{2} \bar{\Psi}_{q, Q} \Gamma_{6}^{\beta} \bar{\Psi}_{p, P} \gamma_{\beta} \frac{m+\hat{k}_{1}-\hat{q}_{1}}{\left(k_{1}-q_{1}\right)^{2}-m^{2}} \hat{\varepsilon}_{1}
\end{gathered}
$$

where inverse denominators of gluon propagators are defined as $\mathcal{D}_{1,2}^{-1}=\left(k_{2}-p_{1,2}-q_{1,2}\right)^{2}$ and $\mathcal{D}_{3,4}^{-1}=\left(p_{1,2}+q_{1,2}\right)^{2}$. Pertubative amplitude $\mathcal{T}\left(p_{1}, p_{2} ; q_{1}, q_{2}\right)$ in Eq. (3) describes production of two $c$-quark and $\bar{c}$-antiquark pairs with the momenta $p_{1,2}$ and $q_{2,1}$ respectively. The formation of observable bound states from quark-antiquark pairs is determined in the quark model by the quasipotential wave functions $\Psi_{J / \psi}(p, P)$ and $\Psi_{J / \psi}(q, Q)$. These wave functions are calculated initially in the meson rest frame and then transformed to the reference frames moving with the four-momenta $P$ and $Q$. As a result we obtain in (12) the following 
expressions for relativistic bound state wave functions which determine the transition of heavy quarks to the bound state:

$$
\begin{array}{r}
\bar{\Psi}_{p, P}=\frac{\bar{\Psi}_{0}^{J / \psi}(\mathbf{p})}{\left[\frac{\epsilon(p)}{m} \frac{\epsilon(p)+m}{2 m}\right]}\left[\frac{\hat{v}_{1}-1}{2}+\hat{v}_{1} \frac{\mathbf{p}^{2}}{2 m(\epsilon(p)+m)}-\frac{\hat{p}}{2 m}\right] \times \\
\hat{\varepsilon}_{P}^{*}\left(P, S_{z}\right)\left(1+\hat{v}_{1}\right)\left[\frac{\hat{v}_{1}+1}{2}+\hat{v}_{1} \frac{\mathbf{p}^{2}}{2 m(\epsilon(p)+m)}+\frac{\hat{p}}{2 m}\right], \\
\bar{\Psi}_{q, Q}=\frac{\bar{\Psi}_{0}^{J / \psi}(\mathbf{q})}{\left[\frac{\epsilon(q)}{m} \frac{\epsilon(q)+m}{2 m}\right]}\left[\frac{\hat{v}_{2}-1}{2}+\hat{v}_{2} \frac{\mathbf{q}^{2}}{2 m(\epsilon(q)+m)}+\frac{\hat{q}}{2 m}\right] \times \\
\hat{\varepsilon}_{Q}^{*}\left(Q, S_{z}\right)\left(1+\hat{v}_{2}\right)\left[\frac{\hat{v}_{2}+1}{2}+\hat{v}_{2} \frac{\mathbf{q}^{2}}{2 m(\epsilon(q)+m)}-\frac{\hat{q}}{2 m}\right] .
\end{array}
$$

Leading order vertex functions in (12) are calculated in the Feynman gauge and can be presented as follows:

$$
\begin{aligned}
& \Gamma_{1}^{\beta}=\hat{\varepsilon}_{1} \frac{m-\hat{k}_{1}+\hat{q}_{2}}{\left(k_{1}-q_{2}\right)^{2}-m^{2}} \gamma^{\beta}-8 \gamma^{\beta} \frac{m+\hat{k}_{1}-\hat{p}_{2}}{\left(k_{1}-p_{2}\right)^{2}-m^{2}} \hat{\varepsilon}_{1}, \\
& \Gamma_{2}^{\beta}=\hat{\varepsilon}_{2} \frac{m-\hat{k}_{2}+\hat{q}_{2}}{\left(k_{2}-q_{2}\right)^{2}-m^{2}} \gamma^{\beta}-8 \gamma^{\beta} \frac{m+\hat{k}_{2}-\hat{p}_{2}}{\left(k_{2}-p_{2}\right)^{2}-m^{2}} \hat{\varepsilon}_{2}, \\
& \Gamma_{3}^{\beta}=\hat{\varepsilon}_{1} \frac{m-\hat{k}_{1}+\hat{q}_{2}}{\left(k_{1}-q_{2}\right)^{2}-m^{2}}\left[\gamma^{\beta} \frac{m+\hat{k}_{2}-\hat{p}_{2}}{\left(k_{2}-p_{2}\right)^{2}-m^{2}} \hat{\varepsilon}_{2}-8 \hat{\varepsilon}_{2} \frac{m-\hat{p}_{1}-\hat{p}_{2}-\hat{q}_{1}}{\left(p_{1}+p_{2}+q_{1}\right)^{2}-m^{2}} \gamma^{\beta}\right]+ \\
& \hat{\varepsilon}_{2} \frac{m-\hat{k}_{2}+\hat{q}_{2}}{\left(k_{2}-q_{2}\right)^{2}-m^{2}}\left[\gamma^{\beta} \frac{m+\hat{k}_{1}-\hat{p}_{2}}{\left(k_{1}-p_{2}\right)^{2}-m^{2}} \hat{\varepsilon}_{1}-8 \hat{\varepsilon}_{1} \frac{m-\hat{p}_{1}-\hat{p}_{2}-\hat{q}_{1}}{\left(p_{1}+p_{2}+q_{1}\right)^{2}-m^{2}} \gamma^{\beta}\right]- \\
& 8 \gamma^{\beta} \frac{m+\hat{p}_{1}+\hat{q}_{1}+\hat{q}_{2}}{\left(p_{1}+q_{1}+q_{2}\right)^{2}-m^{2}}\left[\hat{\varepsilon}_{2} \frac{m+\hat{k}_{1}-\hat{p}_{2}}{\left(k_{1}-p_{2}\right)^{2}-m^{2}} \hat{\varepsilon}_{1}+\hat{\varepsilon}_{1} \frac{m+\hat{k}_{2}-\hat{p}_{2}}{\left(k_{2}-p_{2}\right)^{2}-m^{2}} \hat{\varepsilon}_{2}\right]+ \\
& 18 \gamma_{\alpha}\left[\mathcal{D}_{1} \frac{m-\hat{k}_{1}+\hat{q}_{2}}{\left(k_{1}-q_{2}\right)^{2}-m^{2}} \varepsilon_{1}^{\alpha} \gamma_{\mu} \mathfrak{E}_{2}^{\beta \mu}\left(p_{1}+q_{1}\right)-\mathcal{D}_{1} \frac{m+\hat{k}_{1}-\hat{p}_{2}}{\left(k_{1}-p_{2}\right)^{2}-m^{2}} \hat{\varepsilon}_{1} \mathfrak{E}_{2}^{\beta \alpha}\left(p_{1}+q_{1}\right)+\right. \\
& \left.\mathcal{D}_{2} \frac{m-\hat{k}_{2}+\hat{q}_{2}}{\left(k_{2}-q_{2}\right)^{2}-m^{2}} \varepsilon_{2}^{\alpha} \gamma_{\mu} \mathfrak{E}_{1}^{\beta \mu}\left(p_{1}+q_{1}\right)-\mathcal{D}_{2} \frac{m+\hat{k}_{2}-\hat{p}_{2}}{\left(k_{2}-p_{2}\right)^{2}-m^{2}} \hat{\varepsilon}_{2} \mathfrak{E}_{1}^{\beta \alpha}\left(p_{1}+q_{1}\right)\right], \\
& \Gamma_{4}^{\beta}=\hat{\varepsilon}_{1} \frac{m-\hat{k}_{1}+\hat{p}_{1}}{\left(k_{1}-p_{1}\right)^{2}-m^{2}}\left[\gamma^{\beta} \frac{m+\hat{k}_{2}-\hat{q}_{1}}{\left(k_{2}-q_{1}\right)^{2}-m^{2}} \hat{\varepsilon}_{2}-8 \hat{\varepsilon}_{2} \frac{m-\hat{p}_{2}-\hat{q}_{1}-\hat{q}_{2}}{\left(p_{2}+q_{1}+q_{2}\right)^{2}-m^{2}} \gamma^{\beta}\right]+ \\
& \hat{\varepsilon}_{2} \frac{m-\hat{k}_{2}+\hat{p}_{1}}{\left(k_{2}-p_{1}\right)^{2}-m^{2}}\left[\gamma^{\beta} \frac{m+\hat{k}_{1}-\hat{q}_{1}}{\left(k_{1}-q_{1}\right)^{2}-m^{2}} \hat{\varepsilon}_{1}-8 \hat{\varepsilon}_{1} \frac{m-\hat{p}_{2}-\hat{q}_{1}-\hat{q}_{2}}{\left(p_{2}+q_{1}+q_{2}\right)^{2}-m^{2}} \gamma^{\beta}\right]- \\
& 8 \gamma^{\beta} \frac{m+\hat{p}_{1}+\hat{p}_{2}+\hat{q}_{2}}{\left(p_{1}+p_{2}+q_{2}\right)^{2}-m^{2}}\left[\hat{\varepsilon}_{2} \frac{m+\hat{k}_{1}-\hat{q}_{1}}{\left(k_{1}-q_{1}\right)^{2}-m^{2}} \hat{\varepsilon}_{1}+\hat{\varepsilon}_{1} \frac{m+\hat{k}_{2}-\hat{q}_{1}}{\left(k_{2}-q_{1}\right)^{2}-m^{2}} \hat{\varepsilon}_{2}\right]+ \\
& 18 \gamma_{\alpha}\left[\mathcal{D}_{2} \frac{m-\hat{k}_{1}+\hat{p}_{1}}{\left(k_{1}-p_{1}\right)^{2}-m^{2}} \varepsilon_{1}^{\alpha} \gamma_{\mu} \mathfrak{E}_{2}^{\beta \mu}\left(p_{2}+q_{2}\right)-\mathcal{D}_{2} \frac{m+\hat{k}_{1}-\hat{q}_{1}}{\left(k_{1}-q_{1}\right)^{2}-m^{2}} \hat{\varepsilon}_{1} \mathfrak{E}_{2}^{\beta \alpha}\left(p_{2}+q_{2}\right)+\right.
\end{aligned}
$$




$$
\begin{gathered}
\left.\mathcal{D}_{1} \frac{m-\hat{k}_{2}+\hat{p}_{1}}{\left(k_{2}-p_{1}\right)^{2}-m^{2}} \varepsilon_{2}^{\alpha} \gamma_{\mu} \mathfrak{E}_{1}^{\beta \mu}\left(p_{2}+q_{2}\right)-\mathcal{D}_{1} \frac{m+\hat{k}_{2}-\hat{q}_{1}}{\left(k_{2}-q_{1}\right)^{2}-m^{2}} \hat{\varepsilon}_{2} \mathfrak{E}_{1}^{\beta \alpha}\left(p_{2}+q_{2}\right)\right]+ \\
18 \mathcal{D}_{3} \gamma_{\nu}\left[2 \varepsilon_{1} \varepsilon_{2} g^{\nu \beta}-\varepsilon_{1}^{\nu} \varepsilon_{2}^{\beta}-\varepsilon_{1}^{\beta} \varepsilon_{2}^{\nu}+\mathcal{D}_{2} \mathfrak{F}^{\nu \beta}\left(p_{1}+q_{1}, p_{2}+q_{2}\right)+\mathcal{D}_{1} \mathfrak{F}^{\beta \nu}\left(p_{2}+q_{2}, p_{1}+q_{1}\right)\right], \\
\Gamma_{5}^{\beta}=\gamma^{\beta} \frac{m+\hat{k}_{1}-\hat{p}_{2}}{\left(k_{1}-p_{2}\right)^{2}-m^{2}} \hat{\varepsilon}_{1}-8 \hat{\varepsilon}_{1} \frac{m-\hat{k}_{1}+\hat{q}_{2}}{\left(k_{1}-q_{2}\right)^{2}-m^{2}} \gamma^{\beta}, \\
\Gamma_{6}^{\beta}=\gamma^{\beta} \frac{m+\hat{k}_{2}-\hat{p}_{2}}{\left(k_{2}-p_{2}\right)^{2}-m^{2}} \hat{\varepsilon}_{2}-8 \hat{\varepsilon}_{2} \frac{m-\hat{k}_{2}+\hat{q}_{2}}{\left(k_{2}-q_{2}\right)^{2}-m^{2}} \gamma^{\beta},
\end{gathered}
$$

where we introduce the following tensors:

$$
\begin{gathered}
\mathfrak{E}_{1,2}^{\alpha \beta}(x)=\frac{1}{2}\left(2 x \varepsilon_{1,2} g^{\alpha \beta}-\left(k_{1,2}^{\beta}+x^{\beta}\right) \varepsilon_{1,2}^{\alpha}+\left(2 k_{1,2}^{\alpha}-x^{\alpha}\right) \varepsilon_{1,2}^{\beta}\right), \\
\mathfrak{F}^{\alpha \beta}(x, y)=4\left(x \varepsilon_{1}\right)\left(y \varepsilon_{2}\right) g^{\alpha \beta}+\left(k_{1}+x\right)\left(k_{2}+y\right) \varepsilon_{1}^{\alpha} \varepsilon_{2}^{\beta}+\varepsilon_{1} \varepsilon_{2}\left(2 k_{1}^{\alpha}-x^{\alpha}\right)\left(2 k_{2}^{\beta}-y^{\beta}\right)+ \\
2 x\left(\varepsilon_{1} \varepsilon_{2}^{\alpha}-\varepsilon_{2} \varepsilon_{1}^{\alpha}\right)\left(2 k_{2}^{\beta}-y^{\beta}\right)-2 y\left(\varepsilon_{1} \varepsilon_{2}^{\beta}-\varepsilon_{2} \varepsilon_{1}^{\beta}\right)\left(2 k_{1}^{\alpha}-x^{\alpha}\right)- \\
x \varepsilon_{1}\left(x^{\alpha}+4 y^{\alpha}\right) \varepsilon_{2}^{\beta}-y \varepsilon_{2}\left(4 x^{\beta}+y^{\beta}\right) \varepsilon_{1}^{\alpha} .
\end{gathered}
$$

Our expressions for the amplitude (12) and vertex functions (15) contain relative momenta $p$ and $q$ in exact form. In order to take into account relativistic corrections of the second order in $p$ and $q$ we expand all inverse denominators of the quark and gluon propagators. Such expansions look as follows:

$$
\begin{gathered}
\frac{1}{\left(p_{1}+q_{1}\right)^{2}}=\frac{4}{s}-\frac{16}{s^{2}}\left[(p+q)^{2}+p Q+q P\right]+\cdots, \\
\frac{1}{\left(k_{2}-q_{2}\right)^{2}-m^{2}}=\frac{2}{t-M^{2}}-\frac{4}{\left(t-M^{2}\right)^{2}}\left[q^{2}+2 q k_{2}\right]+\cdots,
\end{gathered}
$$

where the Mandelstam variables for the gluonic subprocess $s$ and $t$ are defined in (5). There are 16 quark and gluon propagators in the amplitude (12) which have to be expanded in the same way as in (17). All denominators of these propagators in nonrelativistic limit take one of the following forms: $\left(t-M^{2}\right) / 2,\left(M^{2}-s-t\right) / 2, \pm s / 4$ or $s / 2$. Then, the inequalities

$$
4 M^{2} \leq s, \quad\left|t+\frac{s}{2}-M^{2}\right| \leq \frac{s}{2} \sqrt{1-\frac{4 M^{2}}{s}}
$$

mean that in the case of the most unfavorable values of the variables $x_{1,2}$ and $t$ we can roughly estimate expansion parameters in (17) as $2 p^{2} / M^{2}$ and $2 q^{2} / M^{2}$. Preserving in the expanded amplitude terms up to the second order in the relative momenta $p$ and $q$, we can perform angular integration using the following relations for $\mathcal{S}$-wave charmonium:

$$
\begin{gathered}
\int \frac{\Psi_{0}^{\mathcal{S}}(\mathbf{p})}{\left[\frac{\epsilon(p)}{m} \frac{\epsilon(p)+m}{2 m}\right]} \frac{d \mathbf{p}}{(2 \pi)^{3}}=\frac{1}{\sqrt{2} \pi} \int_{0}^{\infty} \frac{p^{2} R_{\mathcal{S}}(p)}{\left[\frac{\epsilon(p)}{m} \frac{\epsilon(p)+m}{2 m}\right]} d p \\
\int p_{\mu} p_{\nu} \frac{\Psi_{0}^{\mathcal{S}}(\mathbf{p})}{\left[\frac{\epsilon(p)}{m} \frac{\epsilon(p)+m}{2 m}\right]} \frac{d \mathbf{p}}{(2 \pi)^{3}}=-\frac{1}{3 \sqrt{2} \pi}\left(g_{\mu \nu}-v_{1 \mu} v_{1 \nu}\right) \int_{0}^{\infty} \frac{p^{4} R_{\mathcal{S}}(p)}{\left[\frac{\epsilon(p)}{m} \frac{\epsilon(p)+m}{2 m}\right]} d p
\end{gathered}
$$


where $R_{\mathcal{S}}(p)$ is the radial charmonium wave function.

To illustrate the described transformations we present here the result of the calculation of the first amplitude in Fig. [1:

$$
\begin{aligned}
& \mathcal{M}_{1}^{a b}=\frac{32 \alpha_{s}^{2} \delta^{a b}}{9 m s^{4}} \int \frac{m+\epsilon(p)}{2 \epsilon(p)} R(p) p^{2} \int \frac{m+\epsilon(q)}{2 \epsilon(q)} R(q) q^{2}\left\{3 s ^ { 2 } \left[\varepsilon_{1} \cdot \varepsilon_{2}\left(s \varepsilon_{P}^{*} \cdot \varepsilon_{Q}^{*}-2 \varepsilon_{P}^{*} \cdot Q \varepsilon_{Q}^{*} \cdot P\right)-\right.\right. \\
& 2 \varepsilon_{P}^{*} \cdot \varepsilon_{Q}^{*}\left(\varepsilon_{1} \cdot P \varepsilon_{2} \cdot Q+\varepsilon_{1} \cdot Q \varepsilon_{2} \cdot P\right)+2 \varepsilon_{P}^{*} \cdot Q\left(\varepsilon_{1} \cdot P \varepsilon_{2} \cdot \varepsilon_{Q}^{*}+\varepsilon_{1} \cdot \varepsilon_{Q}^{*} \varepsilon_{2} \cdot P\right)-\varepsilon_{1} \cdot \varepsilon_{P}^{*} \times \\
& \left.\left(s \varepsilon_{2} \cdot \varepsilon_{Q}^{*}-2 \varepsilon_{2} \cdot Q \varepsilon_{Q}^{*} \cdot P\right)-\varepsilon_{2} \cdot \varepsilon_{P}^{*}\left(s \varepsilon_{1} \cdot \varepsilon_{Q}^{*}-2 \varepsilon_{1} \cdot Q \varepsilon_{Q}^{*} \cdot P\right)\right]\left(3\left(1-c_{p}-c_{q}-c_{p}^{2}-c_{q}^{2}\right)+c_{p} c_{q} \times\right. \\
& \left.\left(67+3 c_{p}+3 c_{q}\right)+3 c_{p}^{2} c_{q}^{2}\right)-64 m^{2} s\left[\varepsilon_{P}^{*} \cdot Q\left(\varepsilon_{1} \cdot P \varepsilon_{2} \cdot \varepsilon_{Q}^{*}+\varepsilon_{1} \cdot \varepsilon_{Q}^{*} \varepsilon_{2} \cdot P\right)+\varepsilon_{Q}^{*} \cdot P\left(\varepsilon_{1} \cdot Q \varepsilon_{2} \cdot \varepsilon_{P}^{*}+\right.\right. \\
& \left.\left.\varepsilon_{1} \cdot \varepsilon_{P}^{*} \varepsilon_{2} \cdot Q\right)\right]\left(3\left(c_{p}+c_{q}\right)+c_{p} c_{q}\left(194-3 c_{p}-3 c_{q}\right)\right)+16 m^{2} s^{2}\left[\varepsilon_{1} \cdot \varepsilon_{P}^{*} \varepsilon_{2} \cdot \varepsilon_{Q}^{*}+\varepsilon_{1} \cdot \varepsilon_{Q}^{*} \varepsilon_{2} \cdot \varepsilon_{P}^{*}\right] \times \\
& \left(9\left(c_{p}+c_{q}\right)+c_{p} c_{q}\left(380-9 c_{p}-9 c_{q}\right)\right)+192 m^{2} s \varepsilon_{P}^{*} \cdot \varepsilon_{Q}^{*}\left[\varepsilon_{1} \cdot P \varepsilon_{2} \cdot Q+\varepsilon_{1} \cdot Q \varepsilon_{2} \cdot P\right]\left(c_{p}+c_{q}+\right. \\
& \left.c_{p} c_{q}\left(62-c_{p}-c_{q}\right)\right)+16 m^{2} s \varepsilon_{1} \cdot \varepsilon_{2}\left(\varepsilon _ { P } ^ { * } \cdot \varepsilon _ { Q } ^ { * } \left[32 m^{2}\left(3\left(c_{p}+c_{q}\right)+c_{p} c_{q}\left(329-3 c_{p}-3 c_{q}\right)\right)-3 s \times\right.\right. \\
& \left.\left(3-2 c_{p}-2 c_{q}-3 c_{p}^{2}-3 c_{q}^{2}\right)+s c_{p} c_{q}\left(613+6 c_{p}+6 c_{q}\right)+9 s c_{p}^{2} c_{q}^{2}\right]+4 \varepsilon_{P}^{*} \cdot Q \varepsilon_{Q}^{*} \cdot P\left[3\left(c_{p}+c_{q}\right)+\right. \\
& \left.\left.c_{p} c_{q}\left(202-3 c_{p}-3 c_{q}\right)\right]\right)+512 m^{2} c_{p} c_{q}\left(2 \varepsilon_{P}^{*} \cdot Q \varepsilon_{Q}^{*} \cdot P\left(\varepsilon_{1} \cdot Q \varepsilon_{2} \cdot P+\varepsilon_{1} \cdot P \varepsilon_{2} \cdot Q\right)-2 m^{2} \varepsilon_{P}^{*} \cdot \varepsilon_{Q}^{*} \times\right. \\
& {\left[1064 m^{2} \varepsilon_{1} \cdot \varepsilon_{2}+\varepsilon_{1} \cdot P\left(125 \varepsilon_{2} \cdot Q-8 \varepsilon_{2} \cdot P\right)+\varepsilon_{1} \cdot Q\left(125 \varepsilon_{2} \cdot P-8 \varepsilon_{2} \cdot Q\right)\right]-} \\
& m^{2}\left[266 \varepsilon_{1} \cdot \varepsilon_{2} \varepsilon_{P}^{*} \cdot Q \varepsilon_{Q}^{*} \cdot P+\varepsilon_{1} \cdot \varepsilon_{P}^{*}\left(131 s \varepsilon_{2} \cdot \varepsilon_{Q}^{*}+2 \varepsilon_{Q}^{*} \cdot P\left(4 \varepsilon_{2} \cdot P-129 \varepsilon_{2} \cdot Q\right)\right)+\varepsilon_{1} \cdot \varepsilon_{Q}^{*} \times\right. \\
& \left(131 s \varepsilon_{2} \cdot \varepsilon_{P}^{*}+2 \varepsilon_{P}^{*} \cdot Q\left(4 \varepsilon_{2} \cdot Q-129 \varepsilon_{2} \cdot P\right)\right)+2 \varepsilon_{1} \cdot P\left(4 \varepsilon_{2} \cdot \varepsilon_{P}^{*} \varepsilon_{Q}^{*} \cdot P-129 \varepsilon_{2} \cdot \varepsilon_{Q}^{*} \varepsilon_{P}^{*} \cdot Q\right)+ \\
& \left.\left.\left.2 \varepsilon_{1} \cdot Q\left(4 \varepsilon_{2} \cdot \varepsilon_{Q}^{*} \varepsilon_{P}^{*} \cdot Q-129 \varepsilon_{2} \cdot \varepsilon_{P}^{*} \varepsilon_{Q}^{*} \cdot P\right)\right]\right)\right\} d p d q,
\end{aligned}
$$

where we introduce the relativistic parameter $c_{p}=\frac{m-\epsilon(p)}{m+\epsilon(p)}$. Extracting relativistic factors $(\epsilon+m) / 2 \epsilon$ in the integrals over both relative momenta $\mathbf{p}$ and $\mathbf{q}$ we observe that the amplitude $\mathcal{M}_{1}^{a b}$ is a power-like expansion in relativistic parameters $c_{p}$ and $c_{q}$. Due to the presence of four different polarization vectors, which correspond to incoming gluons and outcoming $J / \psi$ particles, the result (20) appears to be sufficiently lengthy. We have also obtained analogous expressions for remained 30 diagrams, but due to the bulkiness of the total amplitude they are not presented here.

To calculate the cross section we have to sum the squared modulus of the amplitude upon all polarizations using the following relations for final $J / \psi$ mesons and initial gluons correspondingly:

$$
\sum_{\lambda} \varepsilon_{P}^{\mu} \varepsilon_{P}^{* \nu}=v_{1}^{\mu} v_{1}^{\nu}-g^{\mu \nu}, \quad \sum_{\lambda} \varepsilon_{Q}^{\mu} \varepsilon_{Q}^{* \nu}=v_{2}^{\mu} v_{2}^{\nu}-g^{\mu \nu}, \quad \sum_{\lambda} \varepsilon_{1,2}^{\mu} \varepsilon_{1,2}^{* \nu}=\frac{k_{1}^{\mu} k_{2}^{\nu}+k_{1}^{\nu} k_{2}^{\mu}}{k_{1} \cdot k_{2}}-g^{\mu \nu} .
$$

We find it useful to present the differential cross section for double charmonium production in the proton-proton interaction in the following form:

$$
\frac{d \sigma}{d t}[g g \rightarrow J / \psi J / \psi](t, s)=\frac{\pi m^{2} \alpha_{s}^{4}}{2304 s^{2}}|\tilde{R}(0)|^{4} \sum_{i=0}^{3} \omega_{i} F^{(i)}(t, s),
$$


where the function $F^{(0)}$ describes the LO contribution. It coincides with the nonrelativistic analytical expression for the cross section obtained for the studied process in [11, 13, 15, 26]. The functions $F^{(i)}(i=1,2,3)$ describe relativistic corrections. Explicit expressions for all functions $F^{(i)}$ entering the cross section (22) are written in Appendix A. A number of specific parameters $\omega_{i}$ appeared in (22) are defined as

$$
\omega_{0}=1, \quad \omega_{1}=\frac{I_{1}}{I_{0}}, \quad \omega_{2}=\frac{I_{2}}{I_{0}}, \quad \omega_{3}=\omega_{1}^{2} .
$$

They comprise the nonperturbative parameters in the relativistic quark model which determine the transition of quarks and antiquarks into the bound states. The parameter $\tilde{R}(0)$, which represents the relativistic generalization of radial wave function at the origin, is defined by the formula:

$$
\tilde{R}(0)=\sqrt{\frac{2}{\pi}} \int_{0}^{\infty} \frac{m+\epsilon(p)}{2 \epsilon(p)} R(p) p^{2} d p .
$$

The parameters $\omega_{i}$ are determined by integrals containing the bound state wave function in the following form:

$$
I_{0}=\int_{0}^{\infty} \frac{m+\epsilon(p)}{2 \epsilon(p)} R(p) p^{2} d p, \quad I_{1,2}=\int_{0}^{m} \frac{m+\epsilon(p)}{2 \epsilon(p)}\left(\frac{m-\epsilon(p)}{m+\epsilon(p)}\right)^{1,2} R(p) p^{2} d p .
$$

Our basic relations for the cross section (22) evidently show that there exists another source of relativistic corrections connected with the charmonium wave functions. For their calculation with the desired accuracy we suppose that the dynamics of a $c \bar{c}$-pair is determined by the QCD generalization of the standard Breit Hamiltonian [27], which in the c.m. reference frame can be written as

$$
\begin{gathered}
H=H_{0}+\Delta U_{1}+\Delta U_{2}+\Delta U_{3}, \quad H_{0}=2 \sqrt{\mathbf{p}^{2}+m^{2}}-2 m-\frac{C_{F} \tilde{\alpha}_{s}}{r}+A r+B \\
\Delta U_{1}(r)=-\frac{C_{F} \alpha_{s}^{2}}{4 \pi r}\left[2 \beta_{0} \ln (\mu r)+a_{1}+2 \gamma_{E} \beta_{0}\right], \quad a_{1}=\frac{31}{3}-\frac{10}{9} n_{f}, \quad \beta_{0}=11-\frac{2}{3} n_{f} \\
\Delta U_{2}(r)=-\frac{C_{F} \alpha_{s}}{2 m^{2} r}\left[\mathbf{p}^{2}+\frac{\mathbf{r}(\mathbf{r p}) \mathbf{p}}{r^{2}}\right]+\frac{\pi C_{F} \alpha_{s}}{m^{2}} \delta(\mathbf{r})+\frac{3 C_{F} \alpha_{s}}{2 m^{2} r^{3}}(\mathbf{S L})- \\
\frac{C_{F} \alpha_{s}}{2 m^{2}}\left[\frac{\mathbf{S}^{2}}{r^{3}}-3 \frac{(\mathbf{S r})^{2}}{r^{5}}-\frac{4 \pi}{3}\left(2 \mathbf{S}^{2}-3\right) \delta(\mathbf{r})\right]-\frac{C_{A} C_{F} \alpha_{s}^{2}}{2 m r^{2}} \\
\Delta U_{3}(r)=f_{V}\left[\frac{A}{2 m^{2} r}\left(1+\frac{8}{3} \mathbf{S}_{1} \mathbf{S}_{2}\right)+\frac{3 A}{2 m^{2} r} \mathbf{L}+\frac{A}{3 m^{2} r}\left(\frac{3}{r^{2}}\left(\mathbf{S}_{1} \mathbf{r}\right)\left(\mathbf{S}_{2} \mathbf{r}\right)-\mathbf{S}_{1} \mathbf{S}_{2}\right)\right]-\left(1-f_{V}\right) \frac{A}{2 m^{2} r} \mathbf{L S}
\end{gathered}
$$

where $\mathbf{L}=[\mathbf{r} \times \mathbf{p}], \mathbf{S}=\mathbf{S}_{1}+\mathbf{S}_{2}, n_{f}$ is a number of flavors, $C_{A}=3$ and $C_{F}=4 / 3$ are the color factors of the SU(3) color group, $\gamma_{E}$ is the Euler constant. The parameter $f_{V}$ of vector-exchange confining potential was set to be $f_{V}=0.7$. The mass of heavy $c$-quark in our model is equal to $m=1.55 \mathrm{GeV}$. For the dependence of the QCD coupling constant $\tilde{\alpha}_{s}(\mu)$ on the renormalization point $\mu$ in the pure Coulomb term in (26) we use the three-loop result [28]

$$
\begin{gathered}
\tilde{\alpha}_{s}(\mu)=\frac{4 \pi}{\beta_{0} \mathcal{L}}-\frac{4 \pi b_{1} \mathcal{L}}{\left(\beta_{0} \mathcal{L}\right)^{2}}+\frac{4 \pi}{\left(\beta_{0} \mathcal{L}\right)^{3}}\left[b_{1}^{2}\left(\ln ^{2} \mathcal{L}-\ln \mathcal{L}-1\right)+b_{2}\right], \quad \mathcal{L}=\ln \left(\mu^{2} / \Lambda^{2}\right), \\
b_{1}=\frac{64}{9}, \quad b_{2}=\frac{3863}{54}
\end{gathered}
$$


whereas in all other terms of the Hamiltonian (27) we use the one-loop approximation for the coupling constant $\alpha_{s}$. The typical momentum transfer scale in a quarkonium is of order of the quark mass, so we choose the renormalization scale $\mu=m=1.55 \mathrm{GeV}$ and $\Lambda=0.168 \mathrm{GeV}$, which gives $\alpha_{s}=0.314$ for the charmonium states. The parameters of the linear potential $A=0.18 \mathrm{GeV}^{2}$ and $B=-0.16 \mathrm{GeV}$ have the usual values of quark models. Starting with the Hamiltonian (26) we construct the effective potential model based on the Schrödinger equation and find its numerical solutions for $J / \psi$ meson. Additional details of this model are contained in Appendix $\mathrm{C}$ of [18]. Note that the obtained charmonium wave function is strongly decreasing in the region of relativistic momenta $p \gtrsim m$. Our numerical evaluation gives the following values of $\mathcal{S}$-wave charmonium masses: $M_{J / \psi}^{t h}=3.072 \mathrm{GeV}$ and $M_{\eta_{c}}^{t h}=2.988 \mathrm{GeV}$, which lie within the reasonable accuracy in the comparison with their experimentally measured results [29] $M_{J / \psi}^{\text {exp }}=3.097 \mathrm{GeV}$ and $M_{\eta_{c}}^{\text {exp }}=2.980 \mathrm{GeV}$.

\section{NUMERICAL RESULTS AND DISCUSSION}

In this work we investigate the role of relativistic effects in the production of a pair of charmonium mesons in proton-proton interaction in the relativistic quark model. We have studied only the order $\alpha_{s}^{4}$ parton process of gluon-gluon fusion in the color singlet model. At the calculation of the production amplitude (12) we keep relativistic corrections of two types. The first type is determined by several functions depending on the relative quark momenta $\mathbf{p}$ and $\mathbf{q}$ arising from the gluon propagators, the quark propagators, and relativistic meson wave functions. The second type of corrections originates from the perturbative and nonperturbative treatment of the quark-antiquark interaction operator (26) which leads to the essential modification of nonrelativistic wave functions.

For the calculation of relativistic corrections in the bound state wave functions $\Psi_{0}(\mathbf{p})$ we take the Breit potential (27) and construct the effective potential model as in [18, 30] by means of the rationalization of the kinetic energy operator. Using the program of numerical solution of the Schrödinger equation [31] we obtain the following values of all relativistic

parameters entering the cross section (22) : $\tilde{R}(0)=0.57 \mathrm{GeV}^{\frac{3}{2}}, \omega_{1}=-0.051$ and $\omega_{2}=0.0047$.

As it is evident from Eq. (25), our definition of integral parameters $I_{1,2}$ describing relativistic contributions from the production amplitude contains the cutoff at relativistic momentum of order $m$. In spite of the convergence of integrals $I_{1,2}$, our relativistic model can not provide a reliable calculation of the wave functions in the region of relativistic momenta $p \gtrsim m$. So, we introduce a cutoff in (25) in order to avoid possible errors caused by the mentioned uncertainty. It is obviously that in the quark model we can calculate a number of nonperturbative parameters (23) only with certain accuracy. The way of further improvements in the calculation is related in the first place with more accurate construction of the bound state wave function at relativistic momenta. In the approach of NRQCD we encounter analogous difficulties connected with the determination of numerous nonperturbative matrix elements [6].

Let us note also that the cross section (22) contains the fourth power of the modified wave function at the origin $\tilde{R}(0)$ and the strong coupling constant $\alpha_{s}$. Thus, small changes of the bound state wave function can lead to substantial changes in final results for the cross section. The value $\tilde{R}(0)$ is calculated with sufficiently high accuracy with the parameters and potential (26) of the relativistic quark model. The parameter $|\tilde{R}(0)|^{4}$ undergoes essential decrease in comparison with nonrelativistic value. But other relativistic corrections connected 


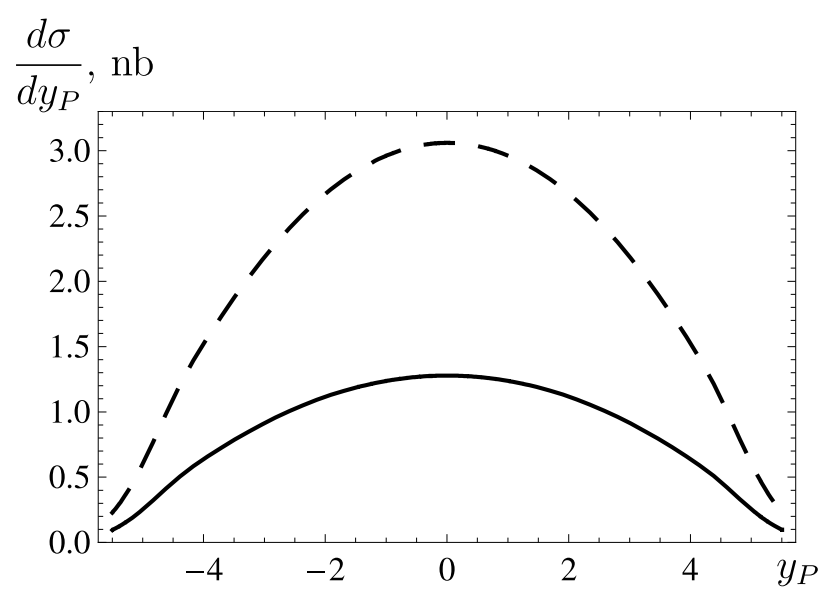

FIG. 4: The differential cross sections for $p p \rightarrow 2 J / \psi+X$ at $\sqrt{S}=7 \mathrm{TeV}$ as functions of rapidity $y_{P}$. Solid and dashed curves represent total and nonrelativistic results respectively.

with the functions $F^{(i)}(i=1,2,3)$ in (22) have the opposite effect on the cross section value (22). Analytical expression of nonrelativistic contribution to the cross section which is determined by $F^{(0)}$ coincides with previous calculations in [11, 13 15, 26]. In the evaluation of $\alpha_{s}$ we set the renormalization scale to be the transverse mass $\mu=m_{T}=\sqrt{4 m^{2}+P_{T}^{2}}$, which is generally accepted choice. For the running coupling constant $\alpha_{s}(\mu)$ we use the LO result with the initial value $\alpha_{s}\left(\mu=M_{z}\right)=0.118$.

The basic expression (2) for the calculation of the differential cross section contains the gluon distribution functions in the proton because the leading contribution comes from a gluon fusion process. When the energy of colliding beams increases, the initial parton momentum fraction $x_{i}$ needed to produce heavy quarkonium decreases. It reaches the region in $x$ where the number of the gluons becomes much larger than the number of quarks. The gluon distribution function determines the probability to find a gluon in the proton with some momentum fraction. There exists a number of the parameterizations for partonic distribution functions [35]. We use the gluon PDF from the set CTEQ5L as in Ref. [11]. The total numerical value of the cross section obtained from (22) is equal to

$$
\sigma_{\text {total }}=9.6 \mathrm{nb}
$$

Due to law-x behavior of gluon distribution functions, the main contribution to the integral cross section (29) results from the region $x_{1,2} \sim 10^{-3}$. To be more precise, this region is determined by the condition: $7.8 \cdot 10^{-7}<x_{1} x_{2}<7.8 \cdot 10^{-6}$. In the frequently used nonrelativistic limit when $\psi(0)=0.21 \mathrm{GeV}^{\frac{3}{2}}$ and $R(0)=0.74 \mathrm{GeV}^{\frac{3}{2}}$ the total value of the cross section amounts $\sigma_{\text {nonrel }}=18.3 \mathrm{nb}$ and agrees with the calculation in [11]. In the nonrelativistic limit of our quark model based on Eqs. (26) and (27) we obtain slightly greater values $R(0)=0.79 \mathrm{GeV}^{\frac{3}{2}}$ and $\sigma_{\text {nonrel }}=23.1 \mathrm{nb}$. To obtain (29) the factorization scale in the parton distribution functions $f_{g / p}(x, \mu)$ is taken equal to the transverse mass too: $\mu=m_{T}$.

To compare the results of our calculation with the measured value of the cross section in [10] it is necessary to write the differential cross section in terms of the rapidity $y_{P}=$ $\frac{1}{2} \ln \frac{P_{0}+P_{\|}}{P_{0}-P_{\|}}$. The rapidities of outcoming charmonia with momenta $P$ and $Q$ can be obtained 

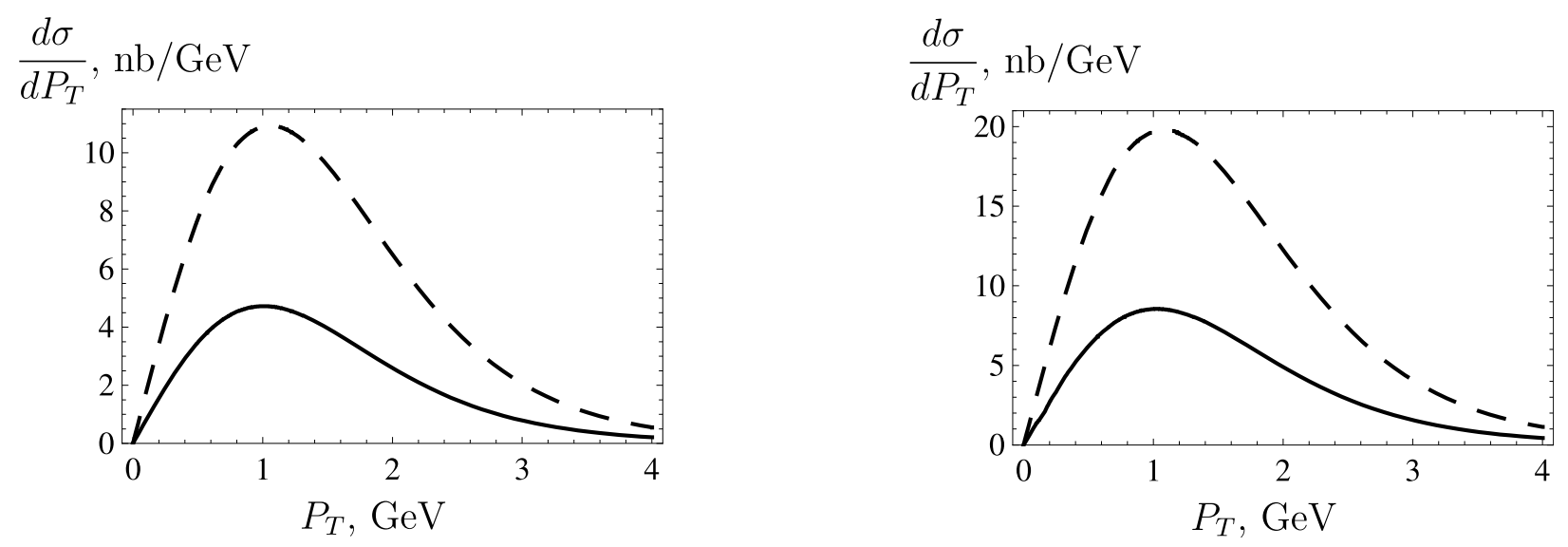

FIG. 5: The differential cross sections for $p p \rightarrow 2 J / \psi+X$ at $\sqrt{S}=7 \mathrm{TeV}$ (left) and $\sqrt{S}=14 \mathrm{TeV}$ (right) as functions of transverse momentum $P_{T}$ of the $J / \psi$ pair integrated over the rapidity. Solid and dashed curves represent total and nonrelativistic results respectively.

in the form:

$$
y_{P, Q}=\frac{1}{2} \ln \frac{x_{1}}{x_{2}} \pm \frac{1}{2} \ln \left[\frac{s}{M^{2}-t}-1\right] .
$$

The differential cross section $d \sigma / d y_{P}$ for the reaction $p p \rightarrow 2 J / \psi+X$ is shown in Fig. 4. It is clear from this plot that relativistic effects strongly influence on the rapidity distribution of the final charmonium. In the $\mathrm{LHCb}$ experiment [10] the rapidity lies in the range $2<y_{P, Q}<4.5$, so we should integrate the differential cross section (2) over rapidities from such interval in order to obtain the value corresponding to the experiment at the $\mathrm{LHCb}$ detector. Then we obtain:

$$
\sigma^{\text {th }}\left(2<y_{P, Q}<4.5\right)=1.6 \mathrm{nb} .
$$

The result (31) is significantly smaller than the experimental value of the cross section (11). Different sources of relativistic corrections in (2) are differently directed. But we observe that combined action of all relativistic effects lead to essential decreasing of the production cross section. In this work we carry out the investigation only of one important source of corrections to the nonrelativistic cross section. Decreasing behavior of the cross section $\sigma(p p \rightarrow 2 J / \psi+X)$ due to the account of relativistic contributions is noticeable clearly in spite of existing theoretical errors occurred in our calculation. In our analysis of the production amplitudes we correctly take into account relativistic contributions of order $O\left(v^{2}\right)$. Therefore the first basic theoretical uncertainty of our calculation is related with the omitted terms of order $O\left(v^{4}\right)$. Since the calculation of the charmonium mass is sufficiently accurate in our model (the error is less then 1\%), we suppose that the uncertainty in the cross section calculation due to omitted relativistic corrections of order $O\left(v^{4}\right)$ in the quark interaction operator (the Breit Hamiltonian) is also very small. Taking into account that the average value of the heavy quark velocity squared in the charmonium is $\left\langle v^{2}\right\rangle=0.3$, we expect that relativistic corrections of order $O\left(v^{4}\right)$ to the cross section (31) coming from the production amplitude should not exceed $30 \%$ of the obtained relativistic result. As we mentioned above in the quasipotential approach we can not find precisely the bound state wave functions in the region of relativistic momenta $p \geq m$. Using indirect arguments related with the mass spectrum calculation we estimate in $10 \%$ the uncertainty in the wave function determination. Larger value of the error will lead to the essential discrepancy between the experiment and 
theory in the calculation of the charmonium mass spectrum. Then the corresponding error in the cross section (31) is not exceeding 20\%. We do not consider a part of theoretical error related with radiative corrections of order $\alpha_{s}$ because these corrections are omitted in our analysis. So, our total theoretical error is not exceeding $36 \%$. To obtain this estimate we add the above mentioned uncertainties in quadrature.

We show in Fig. 5 the distribution over transverse momentum of the $J / \psi$ mesons integrated over all rapidities at $\sqrt{S}=7 \mathrm{TeV}$ (left) and $\sqrt{S}=14 \mathrm{TeV}$ (right). Previous investigations [11, 13, 14] of the pair charmonium production in $p p$ interaction showed that the color singlet channel prevails in the differential cross section $d \sigma / d P_{T}(p p \rightarrow 2 J / \psi+X)$ at small $P_{T}$, but the color octet channel dominates at large $P_{T}$. It can be seen in Fig. 5 at $\sqrt{S}=7 \mathrm{TeV}$ that the account of relativistic corrections leads to the ratio of relativistic and nonrelativistic cross sections $\sigma_{r e l} / \sigma_{n r} \approx 0.4$ near the peak. This trend remains unchanged in the region of high transverse momenta. So, the color-octet contribution retains the dominance at large $P_{T}$. We investigate also the relative value of relativistic corrections in the production rate with the growth of the energy $\sqrt{S}$. Our calculation show that at $\sqrt{S}=14 \mathrm{TeV}$ (see the right plot in Fig. 5) the ratio of relativistic and nonrelativistic cross sections is retained without essential modifications. The cross section increases with the growth of the energy and reaches the value $\sigma^{t h}\left(2<y_{P, Q}<4.5\right)=2.98 \mathrm{nb}$ at $\sqrt{S}=14 \mathrm{TeV}$. It is appropriate to mention here one result regarding to the study of relativistic effects in single $J / \psi$ production at hadron collisions [32]. It was shown in that paper that relativistic corrections to the color-singlet $J / \psi$ hadroproduction of order $O\left(v^{2}\right)$ are at a level of about $1 \%$ for sufficiently large $P_{T}: 5 \leq P_{T} \leq 50 \mathrm{GeV}$. Our calculation demonstrates that in the region of transverse momenta $5 \leq P_{T} \leq 50 \mathrm{GeV}$ the value of relativistic corrections in the cross section of the pair charmonium production reaches $60 \%$. Relativistic corrections which we study in this work include not only the terms of order $O\left(v^{2}\right)$ in the production amplitude but also the same order effects in the long-distance matrix elements. In spite of the difference between (11) and (31), we consider that at present it is difficult to state that there is the discrepancy between the theory and experiment in double charmonium production. Indeed, it is known that NLO in $\alpha_{s}$ contributions have large value in inclusive single-quarkonium production at hadron colliders [4, 33, 34]. The example is found in the inclusive $J / \psi$ production where the NLO corrections to the color-singlet contribution increase the total cross section by a factor of about 2 and the production rate of $J / \psi$ is much increased for larger transverse momentum $P_{T}$. Therefore, one can expect that the NLO corrections to the double charmonium production in proton-proton interaction can smooth the appeared difference between (11) and (31). Moreover, as we mentioned above there exists new mechanism through the double parton scattering which gives the contribution comparable with the standard nonrelativistic result: $\sigma_{D P S}(p p \rightarrow 2 J / \psi+X)=2$ nb [16]. Accounting for this result and our value of the cross section (31) we obtain the summary value $\sigma(p p \rightarrow 2 J / \psi+X)=3.6 \mathrm{nb}$. Then, taking into account the experimental error, the difference with the LHCb experiment does not look so significant.

\section{Acknowledgments}

The authors are grateful to D. Ebert, R.N. Faustov and V.O. Galkin for useful discussions. The work is supported partially by the Ministry of Education and Science of Russian Federation (government order for Samara State U. No. 2.870.2011). 


\section{Appendix A: The coefficients $F^{(i)}$ entering the differential cross section (22)}

$$
\begin{aligned}
& F^{(0)}=\frac{16384}{9 M^{4} s^{6}\left(M^{2}-t\right)^{4}\left(M^{2}-s-t\right)^{4}}\left[7776 M^{24}-432 M^{22}(73 s+216 t)+6 M^{20} \times\right. \\
& \left(9085 s^{2}+60336 s t+85536 t^{2}\right)-16 M^{18}\left(3629 s^{3}+37686 s^{2} t+117855 s t^{2}+106920 t^{3}\right)+ \\
& 2 t^{4}(s+t)^{4}\left(349 s^{4}+2304 s^{3} t+6192 s^{2} t^{2}+7776 s t^{3}+3888 t^{4}\right)+4 M^{16}\left(11927 s^{4}+151588 s^{3} t+\right. \\
& \left.745674 s^{2} t^{2}+1470960 s t^{3}+962280 t^{4}\right)-4 M^{14}\left(7761 s^{5}+109608 s^{4} t+699467 s^{3} t^{2}+\right. \\
& \left.2173908 s^{2} t^{3}+3055320 s t^{4}+1539648 t^{5}\right)+2 M^{12}\left(6952 s^{6}+117893 s^{5} t+897043 s^{4} t^{2}+\right. \\
& \left.3741980 s^{3} t^{3}+8278410 s^{2} t^{4}+8872416 s t^{5}+3592512 t^{6}\right)-4 M^{2} t^{2}(s+t)^{2}\left(9 s^{7}+649 s^{6} t+\right. \\
& \left.6460 s^{5} t^{2}+29630 s^{4} t^{3}+74435 s^{3} t^{4}+105156 s^{2} t^{5}+77868 s t^{6}+23328 t^{7}\right)-2 M^{10}\left(1899 s^{7}+\right. \\
& 43398 s^{6} t+405618 s^{5} t^{2}+2113568 s^{4} t^{3}+6394090 s^{3} t^{4}+10762584 s^{2} t^{5}+9189936 s t^{6}+ \\
& \left.3079296 t^{7}\right)+M^{8}\left(587 s^{8}+19710 s^{7} t+244772 s^{6} t^{2}+1603468 s^{5} t^{3}+6229962 s^{4} t^{4}+\right. \\
& \left.14478304 s^{3} t^{5}+19359816 s^{2} t^{6}+13582080 s t^{7}+3849120 t^{8}\right)-2 M^{6}\left(20 s^{9}+1185 s^{8} t+\right. \\
& 22153 s^{7} t^{2}+193780 s^{6} t^{3}+965358 s^{5} t^{4}+2928368 s^{4} t^{5}+5431786 s^{3} t^{6}+5949528 s^{2} t^{7}+ \\
& \left.3508920 s t^{8}+855360 t^{9}\right)+M^{4}\left(s^{10}+76 s^{9} t+3756 s^{8} t^{2}+52062 s^{7} t^{3}+353472 s^{6} t^{4}+\right. \\
& \left.\left.1398834 s^{5} t^{5}+3421754 s^{4} t^{6}+5210968 s^{3} t^{7}+4784622 s^{2} t^{8}+2414880 s t^{9}+513216 t^{10}\right)\right]
\end{aligned}
$$

$$
\begin{aligned}
& F^{(1)}=-\frac{16384}{27 M^{4} s^{8}\left(M^{2}-t\right)^{5}\left(M^{2}-s-t\right)^{5}}\left[497664 M^{32}-221184 M^{30}(13 s+36 t)+\right. \\
& 5760 M^{28}\left(1285 s^{2}+7680 s t+10368 t^{2}\right)-48 M^{26}\left(243089 s^{3}+2289552 s^{2} t+6612480 s t^{2}+\right.
\end{aligned}
$$$$
\left.5806080 t^{3}\right)+12 M^{24}\left(1090607 s^{4}+13899232 s^{3} t+62988960 s^{2} t^{2}+117411840 s t^{3}+\right.
$$$$
\left.75479040 t^{4}\right)-8 M^{22}\left(1392130 s^{5}+22255745 s^{4} t+136976040 s^{3} t^{2}+399063744 s^{2} t^{3}+\right.
$$$$
\left.540933120 s t^{4}+271724544 t^{5}\right)+8 t^{5}(s+t)^{5}\left(1867 s^{6}+18256 s^{5} t+77728 s^{4} t^{2}+181152 s^{3} t^{3}+\right.
$$$$
\left.246096 s^{2} t^{4}+186624 s t^{5}+62208 t^{6}\right)+4 M^{20}\left(1800338 s^{6}+35626541 s^{5} t+276305481 s^{4} t^{2}+\right.
$$$$
\left.1095702384 s^{3} t^{3}+2313080352 s^{2} t^{4}+2435457024 s t^{5}+996323328 t^{6}\right)-M^{18}\left(3514643 s^{7}+\right.
$$$$
86600280 s^{6} t+825013064 s^{5} t^{2}+4139468480 s^{4} t^{3}+11909795760 s^{3} t^{4}+19466599680 s^{2} t^{5}+
$$$$
\left.16605388800 s t^{6}+5693276160 t^{7}\right)+2 M^{16}\left(659715 s^{8}+19899554 s^{7} t+231809132 s^{6} t^{2}+\right.
$$$$
1426201784 s^{5} t^{3}+5209721940 s^{4} t^{4}+11612866752 s^{3} t^{5}+15333506496 s^{2} t^{6}+
$$$$
\left.10912112640 s t^{7}+3202467840 t^{8}\right)-M^{2} t^{3}(s+t)^{3}\left(664 s^{9}+73857 s^{8} t+969897 s^{7} t^{2}+\right.
$$$$
6006640 s^{6} t^{3}+21622120 s^{5} t^{4}+49033392 s^{4} t^{5}+71696784 s^{3} t^{6}+65938176 s^{2} t^{7}+
$$$$
\left.34725888 s t^{8}+7962624 t^{9}\right)-M^{14}\left(380999 s^{9}+13782634 s^{8} t+195250554 s^{7} t^{2}+\right.
$$$$
1459312784 s^{6} t^{3}+6543590240 s^{5} t^{4}+18571278768 s^{4} t^{5}+33449165568 s^{3} t^{6}+
$$

$\left.36752348160 s^{2} t^{7}+22298664960 s t^{8}+5693276160 t^{9}\right)+2 M^{12}\left(39553 s^{10}+1754120 s^{9} t+\right.$ $30305432 s^{8} t^{2}+275173836 s^{7} t^{3}+1497456520 s^{6} t^{4}+5228976572 s^{5} t^{5}+12018927060 s^{4} t^{6}+$ 
$\left.18013988160 s^{3} t^{7}+16836887232 s^{2} t^{8}+8856207360 s t^{9}+1992646656 t^{10}\right)-4 M^{10} \times$ $\left(2658 s^{11}+152991 s^{10} t+3330028 s^{9} t^{2}+37242554 s^{8} t^{3}+246717845 s^{7} t^{4}+1047413960 s^{6} t^{5}+\right.$ $2970379604 s^{5} t^{6}+5692479912 s^{4} t^{7}+7256996388 s^{3} t^{8}+5868903744 s^{2} t^{9}+2712213504 s t^{10}+$ $\left.543449088 t^{11}\right)+2 M^{4} t(s+t)\left(8 s^{12}+943 s^{11} t+73235 s^{10} t^{2}+1243892 s^{9} t^{3}+10501734 s^{8} t^{4}+\right.$ $53251684 s^{7} t^{5}+176208052 s^{6} t^{6}+396112960 s^{5} t^{7}+612104570 s^{4} t^{8}+641021472 s^{3} t^{9}+$ $\left.434301120 s^{2} t^{10}+171417600 s t^{11}+29859840 t^{12}\right)+2 M^{8}\left(267 s^{12}+31089 s^{11} t+948124 s^{10} t^{2}+\right.$ $13678946 s^{9} t^{3}+112700707 s^{8} t^{4}+584919678 s^{7} t^{5}+2023301960 s^{6} t^{6}+4798139192 s^{5} t^{7}+$ $\left.7834428690 s^{4} t^{8}+8641058880 s^{3} t^{9}+6129541440 s^{2} t^{10}+2515968000 s t^{11}+452874240 t^{12}\right)-$ $M^{6}\left(8 s^{13}+1772 s^{12} t+139013 s^{11} t^{2}+3024912 s^{10} t^{3}+33019131 s^{9} t^{4}+215628282 s^{8} t^{5}+\right.$ $917347710 s^{7} t^{6}+2664532144 s^{6} t^{7}+5399964368 s^{5} t^{8}+7640313480 s^{4} t^{9}+7391197632 s^{3} t^{10}+$ $\left.\left.4650333696 s^{2} t^{11}+1710858240 s t^{12}+278691840 t^{13}\right)\right]$,

$$
F^{(2)}=-4 F^{(0)} \text {, }
$$

$F^{(3)}=\frac{4096}{81 M^{4} s^{10}\left(M^{2}-t\right)^{6}\left(M^{2}-s-t\right)^{6}}\left[31850496 M^{40}-995328 M^{38}(219 s+640 t)+\right.$ $27648 M^{36}\left(33541 s^{2}+153432 s t+218880 t^{2}\right)-9216 M^{34}\left(313031 s^{3}+1832460 s^{2} t+\right.$ $\left.4240836 s t^{2}+3939840 t^{3}\right)+768 M^{32}\left(8244959 s^{4}+63274344 s^{3} t+189539568 s^{2} t^{2}+\right.$ $\left.295052544 s t^{3}+200931840 t^{4}\right)-192 M^{30}\left(52201757 s^{5}+519109072 s^{4} t+2012650416 s^{3} t^{2}+\right.$ $\left.4109435136 s^{2} t^{3}+4827651840 s t^{4}+2571927552 t^{5}\right)+16 M^{28}\left(760108913 s^{6}+9260081144 s^{5} t+\right.$ $46005056832 s^{4} t^{2}+120457679616 s^{3} t^{3}+188334270720 s^{2} t^{4}+177640667136 s t^{5}+$ $\left.77157826560 t^{6}\right)-8 M^{26}\left(1475952353 s^{7}+20899343744 s^{6} t+127598524184 s^{5} t^{2}+\right.$ $423537421056 s^{4} t^{3}+844308839808 s^{3} t^{4}+1076247502848 s^{2} t^{5}+846934050816 s t^{6}+$ $\left.308631306240 t^{7}\right)+16 t^{6}(s+t)^{6}\left(117307 s^{8}+841072 s^{7} t+2960704 s^{6} t^{2}+7010976 s^{5} t^{3}+\right.$ $\left.12425424 s^{4} t^{4}+16277760 s^{3} t^{5}+14715648 s^{2} t^{6}+7962624 s t^{7}+1990656 t^{8}\right)+4 M^{24} \times$ $\left(2269174169 s^{8}+37035895336 s^{7} t+266823310648 s^{6} t^{2}+1087787332416 s^{5} t^{3}+\right.$ $2722434981120 s^{4} t^{4}+4414063320576 s^{3} t^{5}+4767985797120 s^{2} t^{6}+3212405194752 s t^{7}+$ $\left.1003051745280 t^{8}\right)-M^{22}\left(5273945643 s^{9}+102674924584 s^{8} t+863595364448 s^{7} t^{2}+\right.$ $4191387926848 s^{6} t^{3}+12844048277696 s^{5} t^{4}+25933611095040 s^{4} t^{5}+35654616990720 s^{3} t^{6}+$ $\left.33483324506112 s^{2} t^{7}+19674332061696 s t^{8}+5349609308160 t^{9}\right)+2 M^{20}\left(1108300275 s^{10}+\right.$ $26970163113 s^{9} t+270274383346 s^{8} t^{2}+1544514084352 s^{7} t^{3}+5656684280280 s^{6} t^{4}+$ $13921443451712 s^{5} t^{5}+23690831678976 s^{4} t^{6}+28410755383296 s^{3} t^{7}+23612613792768 s^{2} t^{8}+$ $\left.12267586805760 s t^{9}+2942285119488 t^{10}\right)-M^{2} t^{4}(s+t)^{4}\left(82320 s^{11}+10526659 s^{10} t+\right.$ $122742683 s^{9} t^{2}+663347888 s^{8} t^{3}+2199787352 s^{7} t^{4}+5137832384 s^{6} t^{5}+9050135872 s^{5} t^{6}+$ $\left.12183171072 s^{4} t^{7}+12114432000 s^{3} t^{8}+8258236416 s^{2} t^{9}+3403026432 s t^{10}+637009920 t^{11}\right)-$ $M^{18}\left(658318562 s^{11}+20774136911 s^{10} t+256196780079 s^{9} t^{2}+1742958116720 s^{8} t^{3}+\right.$ $7550194930440 s^{7} t^{4}+22215242834368 s^{6} t^{5}+45850626752448 s^{5} t^{6}+67807235088384 s^{4} t^{7}+$ 
$\left.72355739968512 s^{3} t^{8}+53902515363840 s^{2} t^{9}+25023941369856 s t^{10}+5349609308160 t^{11}\right)+$ $4 M^{16}\left(34194446 s^{12}+1432273039 s^{11} t+22383564112 s^{10} t^{2}+185308892030 s^{9} t^{3}+\right.$ $954476169886 s^{8} t^{4}+3325907679280 s^{7} t^{5}+8190596683920 s^{6} t^{6}+14624279688576 s^{5} t^{7}+$ $19220073992064 s^{4} t^{8}+18510299039232 s^{3} t^{9}+12475169980416 s^{2} t^{10}+5218508685312 s t^{11}+$ $\left.1003051745280 t^{12}\right)-M^{14}\left(19246515 s^{13}+1103736798 s^{12} t+22478276084 s^{11} t^{2}+\right.$ $232094334116 s^{10} t^{3}+1443234697510 s^{9} t^{4}+5968097322672 s^{8} t^{5}+17386794613376 s^{7} t^{6}+$ $36925721101440 s^{6} t^{7}+58395873784128 s^{5} t^{8}+69341740385280 s^{4} t^{9}+60881635077120 s^{3} t^{10}+$ $\left.37374891540480 s^{2} t^{11}+14182623756288 s t^{12}+2469050449920 t^{13}\right)+2 M^{4} t^{2}(s+t)^{2} \times$ $\left(1024 s^{14}+151570 s^{13} t+12969265 s^{12} t^{2}+212727487 s^{11} t^{3}+1644135147 s^{10} t^{4}+\right.$ $7651899125 s^{9} t^{5}+24229924530 s^{8} t^{6}+56242255280 s^{7} t^{7}+100001584488 s^{6} t^{8}+$ $138359684800 s^{5} t^{9}+146996284416 s^{4} t^{10}+115115443200 s^{3} t^{11}+61947293184 s^{2} t^{12}+$ $\left.20275826688 s t^{13}+3025797120 t^{14}\right)+2 M^{12}\left(824111 s^{14}+70166611 s^{13} t+1952942482 s^{12} t^{2}+\right.$ $25962244078 s^{11} t^{3}+199207601182 s^{10} t^{4}+988657795894 s^{9} t^{5}+3408779823892 s^{8} t^{6}+$ $8542312029248 s^{7} t^{7}+16016481697464 s^{6} t^{8}+22847237069376 s^{5} t^{9}+24808543913472 s^{4} t^{10}+$ $\left.19996345055232 s^{3} t^{11}+11238532171776 s^{2} t^{12}+3890175787008 s t^{13}+617262612480 t^{14}\right)-$ $M^{6} t(s+t)\left(2176 s^{15}+414368 s^{14} t+34529090 s^{13} t^{2}+736175475 s^{12} t^{3}+7430265423 s^{11} t^{4}+\right.$ $44335186685 s^{10} t^{5}+175863201255 s^{9} t^{6}+499176650440 s^{8} t^{7}+1064569592864 s^{7} t^{8}+$ $1756640077312 s^{6} t^{9}+2262689749056 s^{5} t^{10}+2242313957376 s^{4} t^{11}+1643405663232 s^{3} t^{12}+$ $\left.831421919232 s^{2} t^{13}+256940937216 s t^{14}+36309565440 t^{15}\right)-M^{10}\left(48840 s^{15}+\right.$ $10192723 s^{14} t+435518065 s^{13} t^{2}+7912226162 s^{12} t^{3}+77545065352 s^{11} t^{4}+$ $471337098042 s^{10} t^{5}+1940776757542 s^{9} t^{6}+5736471274304 s^{8} t^{7}+12651167529752 s^{7} t^{8}+$ $21342766010240 s^{6} t^{9}+27846873221696 s^{5} t^{10}+27881390733312 s^{4} t^{11}+$ $\left.20726379168768 s^{3} t^{12}+10705439195136 s^{2} t^{13}+3395959603200 s t^{14}+493810089984 t^{15}\right)+$ $4 M^{8}\left(24 s^{16}+60117 s^{15} t+6480104 s^{14} t^{2}+187031341 s^{13} t^{3}+2509040584 s^{12} t^{4}+\right.$ $19370940805 s^{11} t^{5}+97105255536 s^{10} t^{6}+341150600006 s^{9} t^{7}+884070584925 s^{8} t^{8}+$ $1749157202600 s^{7} t^{9}+2695987058680 s^{6} t^{10}+3249848355136 s^{5} t^{11}+3016059149568 s^{4} t^{12}+$ $\left.\left.2073684017664 s^{3} t^{13}+987205294080 s^{2} t^{14}+288123568128 s t^{15}+38578913280 t^{16}\right)\right]$.

[1] V.V. Kiselev, A.K. Likhoded, S.R. Slabospitsky, and A.V. Tkabladze, Sov. J. Nucl. Phys. 49, 682 (1989).

[2] M. Krämer, Prog. Part. Nucl. Phys. 47, 141 (2001).

[3] E. Braaten, S. Fleming, and T.C. Yuan, Annu. Rev. Nucl. Part. Sci. 46, 197 (1996).

[4] N. Brambilla, S. Eidelman, B.K. Heltsley et al., Eur. Phys. J. C 71, 1534 (2011).

[5] G.T. Bodwin, E. Braaten, and G.P. Lepage, Phys. Rev. D 51, 1125 (1995).

[6] G.T. Bodwin, H.S. Chung, D. Kang, J. Lee, and C. Yu, Phys. Rev. D 77, 094017 (2008); G.T. Bodwin, J. Lee, and C. Yu, Phys. Rev. D 77, 094018 (2008); G.T. Bodwin, D. Kang, 
and J. Lee, Phys. Rev. D 74, 014014 (2006).

[7] K.-Y. Liu, Z.-G. He, and K.-T. Chao, Phys. Rev. D 77, 014002 (2008); Y.-J. Zhang, Y.-Q. Ma, and K.-T. Chao, Phys. Rev. D 78, 054006 (2008); Y.-J. Zhang, Y.-J. Gao, and K.-T. Chao, Phys. Rev. Lett. 96, 092001 (2006).

[8] D. Ebert and A.P. Martynenko, Phys. Rev. D 74, 054008 (2006).

[9] G.V. Pakhlova, P.N. Pakhlov, and S.I. Eidelman, Physics-Uspekhi 53, 219 (2010).

[10] R. Aaij et al. (LHCb Collaboration), Phys. Lett. B 707, 52 (2012).

[11] A.V. Berezhnoy, A.K. Likhoded, A.V. Luchinsky, and A.A. Novoselov, Phys. Rev. D 84, 094023 (2011); A.V. Berezhnoy, A.K. Likhoded, A.V. Luchinsky, and A.A. Novoselov, arXiv:1204.1058[hep-ph].

[12] S.P. Baranov, Phys. Rev. D 84, 054012 (2011).

[13] R. Li, Y.-J. Zhang, and K.-T. Chao, Phys. Rev. D 80, 014020 (2009).

[14] P. Ko, J. Lee, and C. Yu, JHEP01 070, 1 (2011); arXiv:1007.3095[hep-ph].

[15] C.-F. Qiao, L.-P. Sun, and P. Sun, J. Phys. G 37, 075019 (2010); arXiv:0903.0954[hep-ph].

[16] S.P. Baranov, A.M. Snigirev, and N.P. Zotov, Phys. Lett. B 705, 116 (2011).

[17] A.P. Martynenko, Phys. Rev. D 72, 074022 (2005); Phys. Atom. Nucl. 70, 1305 (2007).

[18] D. Ebert, R.N. Faustov, V.O. Galkin, and A.P. Martynenko, Phys. Lett. B 672, 264 (2009); E.N. Elekina and A.P. Martynenko, Phys. Rev. D 81, 054006 (2010); A.P. Martynenko and A.M. Trunin, PoS(QFTHEP2011) 051 (2011).

[19] D. Ebert, R.N. Faustov, V.O. Galkin, and A.P. Martynenko, Phys. Rev. D 70, 014018 (2004).

[20] S.J. Brodsky and J.-P. Lansberg, Phys. Rev. D 81, 051502(R) (2010); J.-P. Lansberg, Int. J. Mod. Phys. A 21, 3857 (2006).

[21] Z. Conesa del Valle, G. Corcella, F. Fleuret et al., Nucl. Phys. B (Proc. Suppl.) 214, 3 (2011).

[22] S.J. Brodsky and J.R. Primack, Ann. Phys. 52, 315 (1969).

[23] R.N. Faustov, Ann. Phys. 78, 176 (1973).

[24] J. Kublbeck, M. Böhm, and A. Denner, Comp. Phys. Comm. 60, 165 (1990); T. Hahn, Comp. Phys. Commun. 140, 418 (2001).

[25] J.A.M. Vermaseren, FORM, arXiv:math-ph/0010025.

[26] C.-F. Qiao, Phys. Rev. D 66, 057504 (2002).

[27] S.N. Gupta, S.F. Radford, and W.W. Repko, Phys. Rev. D 26, 3305 (1982); N. Brambilla, A. Pineda, J. Soto, and A. Vairo, Rev. Mod. Phys. 77, 1423 (2005); K. Melnikov and A. Yelkhovsky, Phys. Rev. D 59, 114009 (1999).

[28] K.G. Chetyrkin, B.A. Kniehl, and M. Steinhauser, Phys. Rev. Lett. 79, 2184 (1997).

[29] K. Nakamura et al. (Particle Data Group), J. Phys. G 37, 075021 (2010).

[30] W. Lucha, F.F. Schöberl, and M. Moser, Preprint HEPHY-PUB 594/93.

[31] W. Lucha and F.F. Schöberl, Int. J. Mod. Phys. C 10, 607 (1999); P. Falkensteiner, H. Grosse, F.F. Schöberl, and P. Hertel, Comp. Phys. Comm. 34, 287 (1985).

[32] Y. Fan, Y.-Q. Ma, and K.-T. Chao, Phys. Rev. D 79, 114009 (2009)

[33] J. Campbell, F. Maltoni, and F. Tramontano, Phys. Rev. Lett. 98, 252002 (2007).

[34] B. Gong and J.-X. Wang, Phys. Rev. Lett. 100, 232001 (2008).

[35] H.L. Lai, J. Huston, S. Kuhlmann, J. Morfin, F. Olness, J.F. Owens, J. Pumplin, and W.K. Tung, Eur. Phys. J. C 12, 375 (2000). 